\title{
Branching Patterns of Medial and Inferior Calcaneal Nerves Around the Tarsal Tunnel
}

\author{
Beom Sulk Kim, MD ${ }^{1}$, Phil Woo Choung, $\mathrm{MD}^{1}$, Soon Wook Kwon, $\mathrm{MD}^{2}$, \\ Im Joo Rhyu, $\mathrm{MD}, \mathrm{PhD}^{2}$, Dong Hwee Kim, $\mathrm{MD}, \mathrm{PhD}^{1}$
}

Departments of ${ }^{1}$ Physical Medicine and Rehabilitation, ${ }^{2}$ Anatomy, Korea University College of Medicine, Seoul, Korea

Objective To demonstrate the bifurcation pattern of the tibial nerve and its branches.

Methods Eleven legs of seven fresh cadavers were dissected. The reference line for the bifurcation point of tibial nerve branches was an imaginary horizontal line passing the tip of the medial malleolus. The distances between the reference line and the bifurcation points were measured. The bifurcation branching patterns were categorized as type I, the pattern in which the medial calcaneal nerve (MCN) branched most proximally; type II, the pattern in which the three branches occurred at the same point; and type III, in which MCN branched most distally.

Results There were seven cases ( $64 \%$ ) of type I, three cases (27\%) of type III, and one case (9\%) of type II. The median MCN branching point was $0.2 \mathrm{~cm}$ (range, -1 to $3 \mathrm{~cm}$ ). The median bifurcation points of the lateral plantar nerves and inferior calcaneal nerves was $-0.6 \mathrm{~cm}$ (range, -1.5 to $1 \mathrm{~cm}$ ) and $-2.5 \mathrm{~cm}$ (range, -3.5 to $-1 \mathrm{~cm}$ ), respectively.

Conclusion MCN originated from the tibial nerve in most cases, and plantar nerves were bifurcated below the medial malleolus. In all cases, inferior calcaneal nerves originated from the lateral plantar nerve. These anatomical findings could be useful for performing procedures, such as nerve block or electrophysiologic studies.

Keywords Tibial nerve, Tarsal tunnel syndrome, Cadaver, Anatomy

\section{INTRODUCTION}

Divisions of the tibial nerve in the tarsal tunnel and the level of bifurcations of the nerve are mentioned in many

Received May 13, 2014; Accepted August 27, 2014

Corresponding author: Dong Hwee Kim

Department of Physical Medicine and Rehabilitation, Korea University Ansan Hospital, 123 Jeokgeum-ro, Danwon-gu, Ansan 425-707, Korea

Tel: +82-31-412-5330, Fax: +82-31-412-5344, E-mail: rmkdh@korea.ac.kr

(c) This is an open-access article distributed under the terms of the Creative Commons Attribution Non-Commercial License (http://creativecommons. org/licenses/by-nc/3.0) which permits unrestricted noncommercial use, distribution, and reproduction in any medium, provided the original work is properly cited.

Copyright (C) 2015 by Korean Academy of Rehabilitation Medicine textbooks and journals as between the medial malleolus and calcaneus [1-5]. However, these studies mainly focused on the branching point of medial plantar nerve and lateral plantar nerve, while the inferior and medial calcaneal nerves were less described. This study was designed to demonstrate the bifurcation pattern of the tibial nerve and the relative location of the tibial nerve branches around the ankle. Thus, we could establish an anatomical guide for electrophysiologic and ultrasonographic evaluation, and treatment of several tarsal region diseases including tarsal tunnel syndrome and isolated branch injury. 


\section{MATERIALS AND METHODS}

Eleven feet obtained from the lower extremities of seven human fresh cadavers were examined in the laboratory of the Department of Anatomy, Korea University College of Medicine. Cadavers with any history of heel

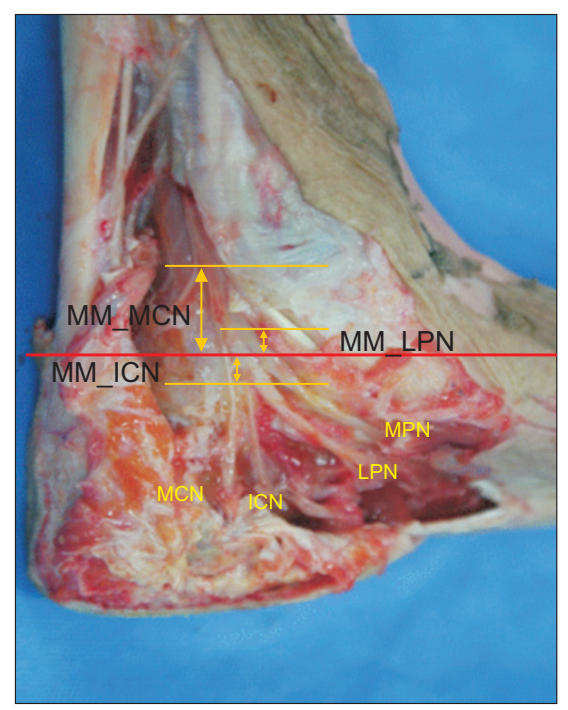

Fig. 1. The distances between the reference line and the bifurcation points were measured. MM, medial malleolus; MCN, medial calcaneal nerve; MPN, medial plantar nerve; LPN, lateral plantar nerve; ICN, inferior calcaneal nerve; MM_MCN, distance between the reference line and the bifurcation point to medial calcaneal nerve; MM_ICN, distance between the reference line and the bifurcation point to inferior calcaneal nerve; MM_LPN, distance between the reference line and the bifurcation point to lateral plantar nerve. or forefoot diseases including tibial arterial diseases or gangrene were excluded. The skin, fascia, and subcutaneous tissue were removed from $20 \mathrm{~cm}$ proximal to the medial malleolus to the plantar surface for exact visualization and measurements. Each foot was placed in the anatomical position with the foot placed perpendicular to the tibia to minimize measurement errors. The tibial nerve and its branches were exposed from the distal leg towards the plantar area. The flexor retinaculum, abductor hallucis muscle, and the plantar aponeurosis were partially removed to expose nerves. The reference line for the bifurcation point of the tibial nerve branches was an imaginary horizontal line that passed the tip of medial malleolus. The distances between the reference line and the bifurcation points were measured. Positive values represented the branching point proximal to the reference line. Negative values represented the branching point distal to the reference line (Fig. 1). The branching pattern of medial calcaneal nerve was categorized into three types. Type I was a pattern in which the medial calcaneal nerve branched proximal to the bifurcation point of medial and lateral plantar nerves. Type II was a branching pattern in which the three branches occurred at the same point. Type III was a pattern in which the medial calcaneal nerve branched distal to the bifurcation point of the medial and lateral plantar nerves (Fig. 2). The branching pattern of the inferior calcaneal nerve and its origin of bifurcation were also noted. Distances were estimated using a measuring tape. Detailed findings were recorded and photographs of anatomic dissection were taken. Mean values of the measurements were statisti-
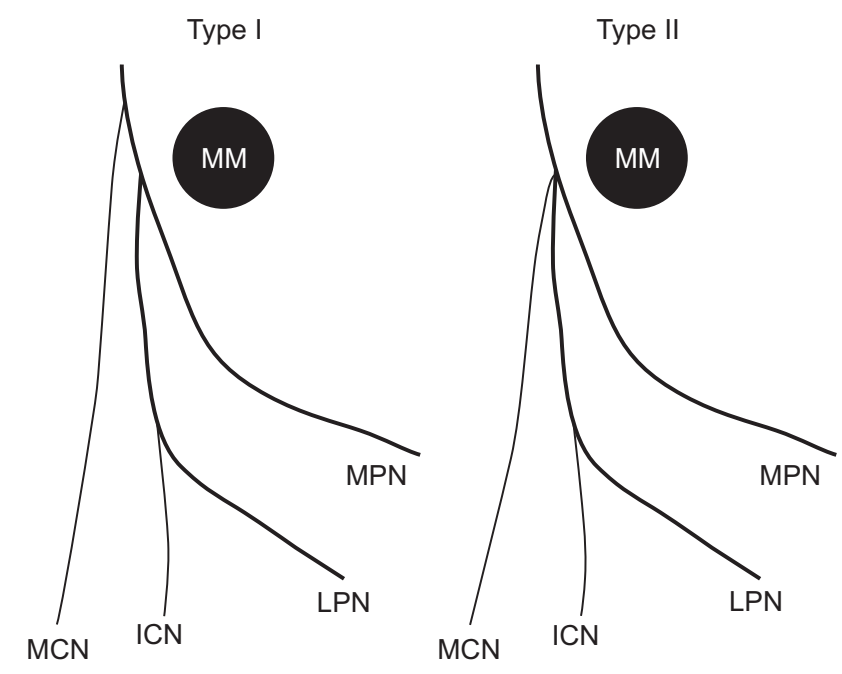

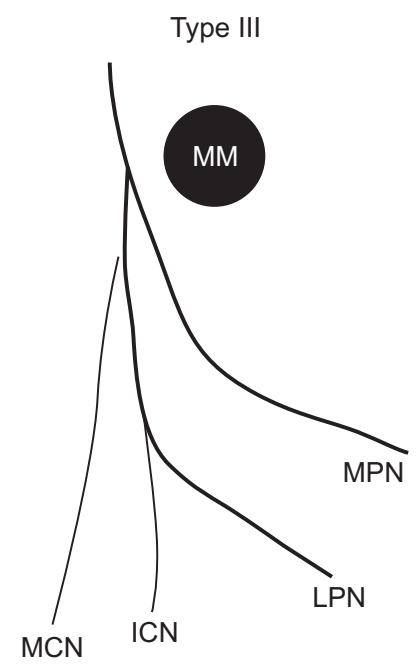

Fig. 2. The branching patterns of tibial nerve were categorized into three types as described in the text. MM, medial malleolus; MCN, medial calcaneal nerve; MPN, medial plantar nerve; LPN, lateral plantar nerve; ICN, inferior calcaneal nerve. 


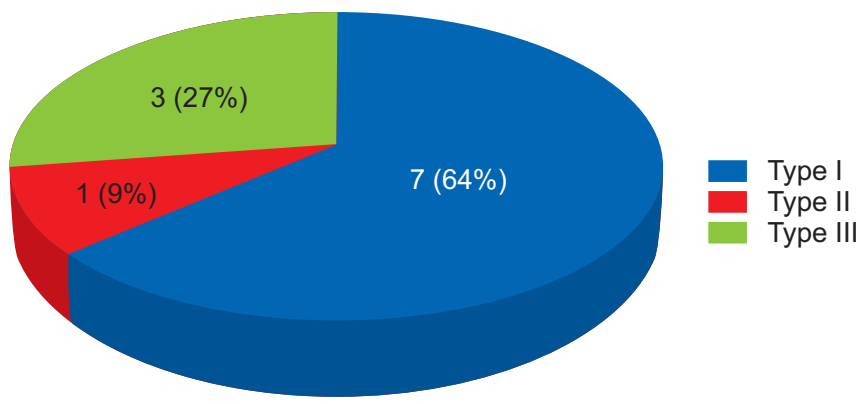

Fig. 3. The bifurcation patterns for tibial nerve. Type I, a pattern in which the medial calcaneal nerve branched proximal to the bifurcation point of medial and lateral plantar nerves; type II, a branching pattern in which the three branches occurred at the same point; type III, a pattern in which the medial calcaneal nerve branched distal to the bifurcation point of the medial and lateral plantar nerves.

cally evaluated.

\section{RESULTS}

The median branching point of medial calcaneal nerve was $0.2 \mathrm{~cm}$ (range, -1 to $3 \mathrm{~cm}$ ). The median bifurcation point of the lateral plantar and inferior calcaneal nerves was $-0.6 \mathrm{~cm}$ (range, -1.5 to $1 \mathrm{~cm}$ ) and $-2.5 \mathrm{~cm}$ (range, -3.5 to $-1 \mathrm{~cm})$, respectively. There were seven cases $(64 \%)$ of type I tibial nerve bifurcation, three cases $(27 \%)$ of type III, and one case (9\%) of type II (Fig. 3).

\section{DISCUSSION}

The tibial nerve is one of the main branches of the sciatic nerve. It passes through the popliteal fossa and branches to posterior calf muscles. The tibial nerve becomes more superficial as it descends along the distal leg. As the bundle reaches the ankle region, it is positioned in a fibro-osseous structure called the tarsal tunnel. This tunnel is bounded by the flexor retinaculum medially, the calcaneus and the talus laterally, and the medial malleolus anteriorly [1-5].

Identification of branching patterns of the tibial nerve around the tarsal tunnel is an important issue in various clinical fields [6-12]. When performing a procedure like external nailing of the tarsal bones or tibial nerve block, clinicians should be aware of branching patterns of the tibial nerve and their relative locations to avoid neural damage.

Horwitz [13] reported that the bifurcation point of the medial and lateral plantar nerves was $1.3 \mathrm{~cm}$ above the tip of the medial malleolus. Dellon and Mackinnon [14] reported that the division was within $2 \mathrm{~cm}$ of the malleolar-calcaneal axis. Bareither et al. [6] indicated that the bifurcation point may extend from $2.8 \mathrm{~cm}$ distal to 14.3 $\mathrm{cm}$ proximal to the tip of the medial malleolus. These studies mainly involved the division of the medial and lateral plantar nerves; our aim was to figure out the bifurcation points of the medial and inferior calcaneal nerves, and their relative location to an easily palpable landmark, the medial malleolus.

Presently, the medial calcaneal nerve originated from the tibial nerve before bifurcation into medial and lateral plantar nerves in all but three cases $(27 \%)$, which arose from the lateral plantar nerve. The origin of medial calcaneal nerve ranged from $1 \mathrm{~cm}$ distal to the tip of the medial malleolus to $3 \mathrm{~cm}$ proximal to the tip. This information about medial calcaneal branching pattern may be useful when performing medial calcaneal nerve block at its origin in patients with plantar fasciitis [7]. Because the medial calcaneal nerve originates from various portions of tibial or lateral plantar nerve, the block site of the nerve might vary from patient to patient. Although ultrasonographic evaluation of the medial calcaneal nerve could be difficult because of its small size, it would be helpful to determine the lesion of the medial calcaneal nerve or the optimal site of blockage in patients with medial calcaneal neuropathy or media plantar fasciitis around the medial ankle.

Far from the medial calcaneal nerve, the inferior calcaneal nerve innervating the abductor digiti minimi muscle originated from the lateral plantar nerve in all cases, in consistent with a previous study [8].

In conclusion, the medial calcaneal nerve is a branch of the posterior tibial nerve or lateral plantar nerve with a wide range of branching points, which might be involved in cases with tarsal tunnel syndrome or isolated injury due to external trauma. It is important to recognize the anatomical characteristics including branching patterns of the tibial nerve around the tarsal tunnel for the accurate diagnosis and the degree of the involvement of the tibial nerve branches in tarsal tunnel syndrome. 


\section{CONFLICT OF INTEREST}

No potential conflict of interest relevant to this article was reported.

\section{REFERENCES}

1. Williams PL, Warwick R, Dyson M, Bannister LH. Gray's anatomy: the anatomical basis of medicine and surgery. 38th ed. Philadelphia: Saunders; 1995.

2. Moore KL, Dalley AF. Clinically oriented anatomy. 4th ed. Philadelphia: Lippincott Williams \& Wilkins; 1999.

3. Draves DJ. Anatomy of the lower extremity. 6 th ed. Baltimore: Williams \& Wilkins; 1986.

4. Ellis H. Clinical anatomy. 8th ed. Oxford: Blackwell Scientific Publications; 1992.

5. Gardner ED, Gray DJ, O'Rahilly R. Anatomy: a regional study of human structure. 3rd ed. Philadelphia: Saunders; 1969.

6. Bareither DJ, Genau JM, Massaro JC. Variation in the division of the tibial nerve: application to nerve blocks. J Foot Surg 1990;29:581-3.

7. Thapa D, Ahuja V. Combination of diagnostic medial calcaneal nerve block followed by pulsed radiofrequency for plantar fascitis pain: a new modality. In- dian J Anaesth 2014;58:183-5.

8. Martin-Oliva X, Elgueta-Grillo J, Veliz-Ayta P, OroscoVillasenor S, Elgueta-Grillo M, Viladot-Perice R. Anatomical variants of the medial calcaneal nerve and the Baxter nerve in the tarsal tunnel. Acta Ortop Mex 2013;27:38-42.

9. Santi MD, Botte MJ. External fixation of the calcaneus and talus: an anatomical study for safe pin insertion. J Orthop Trauma 1996;10:487-91.

10. Casey D, McConnell T, Parekh S, Tornetta P 3rd. Percutaneous pin placement in the medial calcaneus: is anywhere safe? J Orthop Trauma 2002;16:26-9.

11. Mekhail AO, Ebraheim NA, Heck BE, Yeasting RA. Anatomic considerations for safe placement of calcaneal pins. Clin Orthop Relat Res 1996;332:254-9.

12. Greene DL, Thompson MC, Gesink DS, Graves SC. Anatomic study of the medial neurovascular structures in relation to calcaneal osteotomy. Foot Ankle Int 2001;22:569-71.

13. Horwitz MT. Normal anatomy and variations of the peripheral nerves of the leg and foot. Arch Surg 1938;36:626-36.

14. Dellon AL, Mackinnon SE. Tibial nerve branching in the tarsal tunnel. Arch Neurol 1984;41:645-6. 